\title{
1 Genetic basis of transgressive segregation in rice heading phenotypes
}

3 Yohei Koide, ${ }^{*}, 1$ Takashi Uchiyama, ${ }^{*, 1}$ Yuya Ota, ${ }^{* 1}$ Shuntaro Sakaguchi, ${ }^{*}$ Ayumi

4 Tezuka, ${ }^{\dagger}$ Atsushi J. Nagano, ${ }^{\dagger}$ Seiya Ishiguro, ${ }^{*}$ Itsuro Takamure, ${ }^{*}$ and Yuji Kishima*,2

$6 *$ Research Faculty of Agriculture, Hokkaido University, Kita-9 Nishi-9, Kita-ku,

7 Sapporo, 060-8589, Japan

$8 \uparrow$ Faculty of Agriculture, Ryukoku University, 1-5 Yokotani, Seta Oe-cho, Otsu, Shiga

9 520-2194, Japan

$10{ }^{1}$ These authors contributed equally. 
15 Running Title: Rice heading transgressive segregation

17 Key words: rice; transgressive segregation; extreme phenotype; days to heading; QTL

$19{ }^{2}$ Corresponding author: Research Faculty of Agriculture, Hokkaido University, Kita-9

\section{ABSTRACT} are heritably stable. We examined transgressive phenotypes of flowering time in rice.

27 for which the parents had distal DTH, and found very few transgressive phenotypes. 
alleles in A58 and Kitaake, and only the one locus had different alleles. This result indicates that there is a similar genetic basis for DTH between the two varieties. We identified five new quantitative trait loci (QTLs) associated with transgressive DTH phenotypes by genome-wide single nucleotide polymorphism (SNP) analysis. Each of these QTLs showed different degrees of additive effects on DTH, and two QTLs had epistatic effect on each other. These results demonstrated that genome-wide SNP analysis facilitated detection of genetic loci associated with the extreme phenotypes and revealed that the transgressive phenotypes were produced by exchanging complementary alleles of a few minor QTLs in the similar parental genotypes.

\section{INTRODUCTION}

The range of phenotypic variation in a quantitative trait depends on its genetic complexity (Alonso-Blanco And MendeZ-Vigo 2014; Huang AND Han 2014). Cross hybridizations often produce progenies with wider phenotypic variation than their parents, which is referred to as transgressive segregation (RICK AND SMITH 1953; HARLAN 1976; DE ViCENTE AND TANKSLEY 1993). Unlike heterosis, the extreme phenotypes that occur as a result of transgressive segregation can be fixed after the second filial generation (F2). Such extreme phenotypes can have important roles in 
51 evolution (RIESEBERG et al. 2002; DitTRICH-REED AND FitZPATRICK 2013). From a

52 breeding perspective, this phenomenon has also strongly contributed to crop and animal

53 improvements (Vega And Frey 1980; TANKSLEY AND MCCOUCH 1997). However,

54 little is known about the genetic basis of transgressive segregations, which are

55 associated with phenotypic improvement of useful traits in crops.

Days to heading (DTH) determines the regional adaptability of rice (Oryza sativa L.), an important agronomic trait that controls flowering time in rice. Flowering time is a complicated trait in many crops, and the genetic basis of DTH has been well studied in

61 rice; to date, 14 quantitative trait loci (QTLs) were identified based on natural variation and isolated by map-based cloning strategies (EBANA et al. 2011; HoRI et al. 2016; BRAMBILla et al. 2017). We previously examined DTH in rice using six different F2 populations derived from crosses between Kokusyokuto-2 (a Hokkaido landrace denoted as A58) with a short DTH (81 days) used as the seed parent and six varieties 
69 Kasalath F2 population; only this F2 population had some individuals with shorter DTH

70 relative to those of the parents, and the other five F2 populations did not exhibit such

71 extreme phenotypes. In the plants with shorter DTH, we identified a genetic interaction

72 (Ghd7 from A58 and Ehdl from Kasalath) that contributed to the extreme phenotypes

73 produced by the cross of the distantly related parents (OTA et al. 2014).

75 Here, we were interested in determining how the range of phenotypic variation is

76 produced and whether extreme phenotypes can be produced when both parents used in

77 the cross have proximal phenotypes. The progenies from parents with the same

78 genotypes would have very narrow phenotypic variation, while certain range of the

79 phenotypic variation like transgressive phenotypes should be expected in the F2

80 generation from parents with different genotypes that could coincidentally cause similar

81 phenotypes. By testing these predictions, we may be able to identify the unknown genetic entities that produce extreme phenotypes.

84 We specifically focused on phenotypic variations in DTH of a population derived from a cross between two closely related varieties, A58 and Kitaake (an improved variety), 
Kitaake progenies had extreme short or long DTH phenotypes relative to the parents. nucleotide polymorphism (SNP) analysis to detect unknown QTLs associated with extremely short or long DTH. The results obtained here demonstrated that a relatively small number of minor QTLs and their epistatic interactions produced transgressive segregation in DTH. Important genetic properties of the extreme heading phenotypes caused by transgressive segregation are discussed.

\section{MATERIALS AND METHODS}

\section{Genetic stocks}

A rice landrace from Hokkaido, A58, and an improved variety of Hokkaido, Kitaake, were used as parents. A58 seeds were obtained from seed stocks at the Plant Breeding Laboratory in Hokkaido University. Kitaake seeds were obtained from the genebank at the Agricultural Research Department of Hokkaido Research Organization. A58 was 
crossed with Kitaake to obtain F1 seeds. A total of 248 F2 plants were obtained from

self-pollination of the F1 plants. From the 248 F2 plants, 132 were randomly selected to

obtain F3 populations. These F3 populations were used for genetic analysis of DTH

using DNA markers in the $H d l$ locus, which is a major locus that affects DTH in rice

111 individuals that showed late DTH and had a fixed $H d l$ genotype were selected as

112 late-heading populations. Plants in these two selected populations were self-pollinated

113 to produce F4 lines. The genotypes of the 15 early and 15 late lines in the F4 generation

114 were determined by genome-wide SNP analysis (Figure S1).

\section{DTH analysis}

117 Plants were grown in an experimental paddy field at Hokkaido University, Sapporo,

118 Japan (43.1 N). For F2 and F3 populations, DTH was measured in 2013 and 2014,

119 respectively. DTH for the F4 generation was measured in 2015 and 2016 as the number

120 of days from sowing to emergence of the first panicle of a plant. Average DTH of the

121 F3 and F4 populations were calculated from the values of five or six plants. 


\section{Genotyping and sequencing}

124 Genomic DNA was extracted from leaf samples using Plant DNAzol (Invitrogen,

125 Carlsbad, CA, USA). To genotype the $H d 1$ locus, two primers, Hd1L (5'-CGA CGT

126 GCA GGT GTA CTC CG-3') and Hd1R (5'-AAT CTG TGT AAG CAC TG ACG-3'),

127 were used based on the Hd1 sequence. Genome-wide SNPs were detected by double

128 digest restriction site-associated DNA sequencing (ddRAD-Seq) (BAIRD et al. 2008;

129 PETERSON et al. 2012), which began with DNA library preparation using the restriction

130 enzymes $B g l \mathrm{II}$ and EcoRI. Sequencing was performed with $51 \mathrm{bp}$ single-end reads in

131 one lane of a HiSeq2000 Sequencer (Illumina, San Diego, CA, USA) by Macrogen

132 (Seoul, South Korea). The ddRAD-sequencing reads were trimmed with Trimmomatic

133 ver 0.33 (BOLGER et al. 2014) with the following parameters: LEADING:19

134 TRAILING:19 SLIDINGWINDOW:30:20 AVGQUAL:20 MINLEN:51. The trimmed

135 reads were mapped to a RAD reference for the Os-Nipponbare-Reference-IRGSP-1.0

136 using Bowtie 2 (LANGMEAD AND SALZBERG 2012) with a default parameter setting. To

137 build RAD loci, we used the ref_map.pl pipeline in Stacks ver. 1.29 (CATCHEN et al.

138 2011). All RAD-Seq procedures were carried out by Clockmics, Inc. (Izumi, Osaka,

139 Japan). A total of 1,402 SNPs between parental varieties were detected by ddRAD-Seq; among these SNPs, 634 were considered reliable after filtering SNPs that appeared in 
more than $80 \%$ of $\mathrm{F} 4$ plants.

PCR amplicons for the four previously identified genes involved in DTH (Hdl, and PCR Clean-up kit (Macherey-Nagel, Düren, Germany). Purified samples were sequenced in both directions using a Big Dye Terminator Cycle Sequencing kit (Applied Biosystems, Foster City, CA, USA) on an ABI310 automatic sequencer (Applied Biosystems). Sequence alignment was performed using CLUSTAL W 2.1

149 (THOMPSON et al. 1994). The primers used for sequencing the four genes were as follows: Se1/Hd1 [5'-CGA CGT GCA GGT GTA CTC CG-3' and 5'-AAT CTG TGT and $5^{\prime}$-GCC ATC GCG TAG GTA GGT AG-3'], E1/Hd4/Ghd [5'-GCT GGC TGG ACT TCA CTA CC-3' and 5'-CAT GGG CCA CTT CTA AGA TCA-3'], and Hd5/DTH8 [5'-CGG AGT TCA TCA GCT TCG TT-3' and 5'-TGA CCA TGG TGT AAG CAC TG ACG-3'], Hd2/OsPRR37 [5'-TCT TTC TGA TGG CTG TCT GC-3' GAG TGT

\section{Transgressive index}

We defined the transgressive index, which indicates the proportion of phenotypic 

the F2 population by the parental DTH difference.

164 Allelic effects of each of the six loci that influenced DTH of the A58 $\times$ Kitaake hybrid

165 progenies were evaluated as marker genotype values (GODDARD AND HAYES 2007).

166 Average DTH for each allele was calculated based on DTH data collected from all

167 homozygous alleles in the $30 \mathrm{~F} 4$ population lines from 2016 . Then, the central value

168 was determined based on the two phenotypic averages obtained from each of both

169 alleles at the same locus. The marker genotype value was equivalent to the difference

170 between the central value and either allelic average DTH in the same locus.

173 All genetic stocks and sequence data are available on request. Sequence data for this study were deposited in DDBJ (DDBJ accession number XXXXX). 
(Figure 1A), and the earliest plant DTH was equivalent to that of an extreme was not observed in the previously published crosses. 
195 late-heading F3 plants and developed two F4 populations (early and late) by

196 self-pollination. Average DTH of early- and late-heading F4 populations were $63.8 \pm$

$197 \quad 1.32$ and $74.6 \pm 0.99$, respectively, in 2015 , and $72.2 \pm 1.32$ and $80.0 \pm 1.00$,

198 respectively, in 2016 (Figure 2). These differences between DTH of the early and late

199 populations were significant (t-test, $\mathrm{P}<0.001$ ) throughout 2015 and 2016, and it was

200 predicted that these two distinct populations were generated by new genetic interactions

201 derived from the A58 $\times$ Kitaake cross.

\section{Sequence analysis of genes that control DTH, and the effect of Hd1 on DTH}

204 Four loci (E1/Hd4/Ghd7, Hd2/OsPRR37, Se1/Hd1, and Hd5/DTH8) control DTH in

205 varieties from Hokkaido, and their specific alleles facilitated adaptation by producing

206 photoperiod-insensitive varieties with short DTH (ICHITANI et al. 1997; FUJINO AND

207 Sekiguchi 2005a; FujINO AND SeKiguchi 2005b; NonOue et al. 2008; FujINO et al.

208 2013; KoO et al. 2013). To confirm whether these four loci are related to the DTH

209 differences observed in the A58 $\times$ Kitaake F2 population, we compared nucleotide

210 sequences of these loci (Figure 3). Sequence analysis of $H d 1$ showed the presence of

211 polymorphisms, including a 312-bp insertion/deletion in A58 and Kitaake. This 
population. In contrast to $H d 1$, no polymorphisms were detected in the other three loci (E1/Hd4/Ghd7, Hd2/OsPRR37, and Hd5/DTH8) in A58 and Kitaake (Figure 3).

219 showed that $H d l$ had a significant but small effect on DTH in this population $(\mathrm{P}<$

220 0.001), which revealed that the extremely early phenotype of progenies was not fully

221 explainable by only $H d 1$, and another factor(s) may be involved.

\section{Detection of SNPs associated with extreme DTH phenotypes}

224 If a QTL for DTH was located near an SNP, the SNP alleles tended to be associated

225 with early- or late-heading populations. Genome-wide SNP analysis using ddRAD-Seq

226 provided us a total of 634 reliable SNPs for 15 early and 15 late lines in the F4

227 populations (Figure 4). Among the 634 SNPs, 27 were detected as loci where the

228 frequency of either the A58- or Kitaake-type allele was distorted in early- or

229 late-heading populations (Table 2). Of these possible DTH phenotype-related SNPs, we 
231 Figure 4); these SNP clusters represented the chromosomal regions where QTLs for

232 DTH were present.

Validation of relationships between SNP genotypes and DTH

235 Table 2 shows average DTH between two alleles of each of the five SNP clusters in the

$236 \quad$ F4 lines examined in 2015 and 2016. Significant differences $(\mathrm{P}<0.001)$ in DTH

237 between A58- and Kitaake-type homozygous alleles were observed in SNPs on Ch 4 in

238 both 2015 and 2016 (Table 2 and Figure S3). In the SNP cluster on Ch 10, significant

239 differences in DTH between the two alleles were also observed, although the difference

240 was small in 2016. SNPs on Chs 1 and 6 weakly significantly differed between the two

241 alleles that were only observed in 2015. Among the five clusters, the weakest effect was

242 detected in the $\mathrm{Ch} 2$ cluster, which was not significant $(\mathrm{P}=0.14)$, but still clearly

243 discriminated the two alleles by 3 to 4 days. Overall, the order of the five SNP clusters

244 based on additive effects on DTH was: $\mathrm{Ch} 4>(H d l)>10>6>1>2$. The

245 Kitaake-derived alleles in the SNPs on Chs 4, 10, and 6 produced shorter DTH than the

246 A58-derived alleles; alternatively, the A58-derived alleles on Chs 1 and 2 produced

247 shorter DTH than the Kitaake-derived alleles. 
249 Among the selected chromosomal regions (Chs 1, 4, 6, and 10), genetic interactions

250 were tested using the F2 population (Figure 1). Among several combinations of possible

251 epistatic interactions, a strong genetic interaction was identified in the SNPs on Chs 1

252 and 10 (Figures 5 and S4). The A58-derived alleles in the SNPs on Ch1 decreased DTH

253 when they were combined with Kitaake-derived alleles in SNPs on Ch 10, but increased

254 DTH when combined with A58-derived alleles in SNPs on Ch 10 (Figure 5). No known

255 genes associated with DTH are located around these two chromosomal regions. These

256 findings revealed that unknown genes from A58 and Kitaake caused epistatic

257 interactions responsible for the transgressive early phenotype.

Loci weighted by marker genotype values based on DTH data from 2016 are shown in

Figure 6. Among a total of $30 \mathrm{~F} 4$ lines, nine harbored homozygous alleles at all six loci

261 (the five QTLs and Hd1). These nine lines were sorted by DTH (Figure 6). Based on marker genotype values, direction of allelic effect, and the numbers of the alleles with an effect, the order based on DTH was reasonable, although it was not identical to the expectation, because of possible genetic interactions or unknown genes. The short DTH lines tended to have more alleles with a short DTH effect, whereas the long DTH lines contained more alleles with the opposite effect. Therefore, the extreme phenotypes 
produced by transgressive segregation might be defined by allelic composition with different phenotypic effects occurring in either direction.

\section{DISCUSSION}

271 Here, we showed that transgressive segregation occurred in the hybrid progenies of two

272 rice varieties, A58 and Kitaake, both of which have short DTH as an adaptation to

273 high-latitude region. Phenotypic variation beyond the parental range was observed in

274 this segregating population and facilitated uncovering of the genetic basis of

275 transgressive segregation and extreme DTH phenotypes. The two parental varieties

276 shared the same genotypes for three known major QTLs $(E 1 / H d 4 / G h d 7, H d 2 / O s P R R 37$,

277 and $H d 5 / D T H 8$ ) (Figure 3), but different alleles for Sel/Hd1 and several unknown

278 minor QTLs. Such different genotypes in minor QTLs produced new genetic

279 combinations that resulted in transgressive phenotypes of the progenies. QTLs direct

280 either positive or negative actions based on the effect of parental alleles. If negative

281 QTL alleles in either parent are replaced by the positive alleles of the other parent, the

282 progeny could obtain the desired phenotype because of the presence of more positive

283 alleles (DE VICENTE AND TANKSLEY 1993; RIESEBERG et al. 1999). Our results appeared

to demonstrate this scenario, because we observed allelic complementation at QTLs, 
and indicate the importance of such "hidden" genetic variations despite close genetic relationships (HAGIWARA et al. 2006).

We employed SNP analysis with deep sequencing to obtain a sufficient number of markers for the closely related varieties. This was a powerful approach that detected more than 600 genome-wide SNPs between both Hokkaido-adapted varieties (Figure 4).

291 In addition, such similar genetic backgrounds of the two varieties, A58 and Kitaake, 292 facilitated identification of the minor QTLs that shape transgressive early heading by 293 genome-wide SNP analysis.

Our analysis detected five SNP clusters that corresponded to QTLs and the Hdl locus, which contributed to DTH differences in the A58 $\times$ Kitaake progenies (Table 1 and 2). These QTLs were involved in both the additive and epistatic effects on extreme heading phenotypes (Table 2 and Figure 5). Among the SNP clusters, the strongest effect was explained by the Ch 4 cluster, in which the Kitaake-derived allele(s) caused decreased where only one gene, Rice FLO-LFY homolog (RFL) (KYOZUKA et al. 1998), is functionally characterized as a flowering-related gene by the QTL Annotation Rice 
303 Online (Q-TARO) database (http://qtaro.abr.affrc.go.jp/). Similarly, the Ch 6 cluster

304 (from 24.5 to $25.5 \mathrm{Mb}$ ) included a gene for photoperiod sensitivity, Se5 (IzAWA et al.

305 2000). However, no functional polymorphisms in A58 and Kitaake were detected in

306 either RFL or Se5. In the other QTLs found in the SNP clusters on Chs 1, 2, and 10, no

307 known DTH-related genes were identified. These results demonstrated that some

308 unknown genes present in these SNP clusters affected DTH of the Hokkaido varieties.

309 Interestingly, our analysis also showed possible epistatic interactions between genes in

310 SNP clusters on Chs 1 and 10 that shortened DTH (Figure 5). It was previously thought

311 that epistasis is unlikely to be a major cause of transgressive phenotypes (DE VICENTE

312 AND TANKSLEY 1993; RIESEBERG et al. 1999); however, in our study, epistatic

313 interactions explained the transgressive phenotypes observed in the segregating

314 populations (Figure 5).

316 To date, four genes (E1/Hd4/Ghd7, Hd2/OsPRR37, Se1/Hd1, and Hd5/DTH8) were

317 reported to control DTH in improved rice varieties in Hokkaido (ICHITANI et al. 1997;

318 Fujino And SeKiguchi 2005a; FujInO And SeKiguchi 2005b; NonOuE et al. 2008;

319 FuJino et al. 2013; Koo et al. 2013). Among the four genes, loss of functional alleles in

E1/Hd4/Ghd7 and $H d 2 / O s P R R 37$ are necessary to obtain photoperiod insensitivity in 
rice varieties in northern areas (FUJINO AND SEKIGUCHI 2005a; FUJINO AND SEKIGUCHI and $H d 5 / D T H 8$ ) have small effects on photoperiod insensitivity among the varieties in varieties had the same loss-of-function alleles in the E1/Hd4/Ghd7 and Hd2/OsPRR37 loci and had the same functional allele in the Hd5/DTH8 locus (Figure 3). These results indicated that the improved varieties in Hokkaido might have inherited the same E1/Hd4/Ghd7, Hd2/OsPRR37, and Hd5/DTH8 alleles from a landrace similar to A58, which facilitated adaptation, because photoperiod insensitivity was essential for adaptation. In addition to these four loci, newly identified minor QTLs were identified for DTH on Chs 1, 2, 4, 6 and 10 (Table 2 and Figure 4). These QTLs likely contribute to extreme phenotypes of short and long DTH produced by transgressive segregation based on the composition of their complementary alleles (Figure 6). RIESEBERG et al. (1999) made several predictions regarding the cause of transgressive segregation, one of which was consistent with our results: transgressive segregation 
would likely be observed in the F2 population of parents with more proximal

phenotypes (Figure 7). Among the known alleles at the four known loci that are necessary for DTH adaptation to Hokkaido, A58 and Kitaake shared the same alleles at three loci, but not $H d 1$, which indicates that these two varieties possess a considerable amount of common alleles that shorten DTH. Our results demonstrate that transgressive segregation mainly occurred as a result of a few unknown QTLs, in which alleles combined in a complementary manner (Figure 7).

According to RIESEBERG et al. (1999), transgressive segregation tends to occur more frequently in intraspecific crosses, inbred populations, and domesticated populations compared with interspecific crosses, outbred populations, and wild populations, respectively. The lack of a strong positive correlation was observed between parental genetic divergence and transgression frequency (RIESEBERG et al. 1999; RIESEBERG et al. 2003). Our previous study (ОтА et al. 2014) showed that the hybrid progenies of two varieties with distal DTH adapted to different environments exhibited few instances of new allelic combinations were generated in the hybrid progenies (Figure 7). Such 
complex allelic combinations might generate positive and negative genetic interactions,

and offset allelic effects. Stochastically, if there is a large number of segregating loci,

359 individuals rarely accumulate only the alleles with positive effects, but most usually

360 contain alleles with negative effects (Figure 7).

362 This study showed that a few genes and their combinations expanded variation of the

363 DTH phenotype despite similar genetic backgrounds. Consequently, it might be useful

364 to identify QTLs or allelic interactions associated with the transgressive DTH

365 phenotypes in progenies of other varieties with proximal phenotypes. Similarly, to

366 integrate other transgressive phenotypes into breeding programs, alleles with additive

367 effects of minor QTLs should be targeted in varieties with proximal phenotypes.

\section{ACKNOWLEDGMENTS}

370 We are grateful to Ms. K. Aoyama (Lab. Plant Breeding, Hokkaido University) for her

371 help conducting this study. Kitaake seeds were kindly supplied by the Agricultural

372 Research Department of Hokkaido Research Organization. This work was supported by

373 Takano Life Science Research Foundation and JSPS KAKENHI Grant Number 
375 Generation of the Consortium Office for Fostering of Researchers in Future Generations

376 (COFRe), Hokkaido University (to Y. Koide). We thank Mallory Eckstut, $\mathrm{PhD}$, from

377 Edanz Group (www.edanzediting.com/ac) for editing a draft of this manuscript. 
$380 \quad$ Figure 1 Transgressive segregation and genetic relationships between parental varieties.

381 (A) Frequency distribution of DTH from A58 $\times$ Kitaake F2 plants. The transgressive 382 index represents the ratio of the of the F2 population DTH distribution to the parental 383 difference. The DTH difference between A58 and Kitaake was 0.7 days, and the DTH 384 range in the F2 population was 18.0 days, which resulted in a transgressive index of 385 25.7. Standard DTH values of 11 varieties in Hokkaido are indicated by gray arrowheads: (a) Kitaibuki, (b) Hakucho-mochi, (c) Daichinohoshi (d) Hatsushizuku, (e)

387 Hoshinoyume, (f) Kuiku180, (g) Hokuiku-mochi, (h) Nanatsuboshi, (i) Kirara397, (j) 388 Hoshimaru, and (k) Gimpu. (B) Transgressive indexes of crosses between A58 and each 389 of five other varieties. The phylogenetic relationships and the associated dendrogram 390 for the five O. sativa varieties, Nipponbare (japonica), T65 (japonica), IR36 (indica), $391 \quad$ \#108 (indica), and Kasalath (indica, Aus), are presented based on information provided 392 in TAKATA et al. (2005). To calculate the transgressive index, DTH of parental varieties 393 and F2 plants were calculated based on data from OTA et al. (2014).

Figure 2 Frequency distribution of DTH in early- and late-heading F4 lines derived from the A58 × Kitaake cross in 2015 and 2016 . 
397 DTH of the 15 early- and 15 late-heading F4 lines selected in the F3 population was

398 examined in the two years, 2015 and 2016. Early- and late-heading lines are indicated

399 by white and black, respectively. Kitaake and A58 DTH are indicated by white and

400 black arrowheads, respectively, with bars indicating S. E.

402 Figure 3 Comparisons of partial nucleotide sequences from Nipponbare, A58, and

403 Kitaake for the four major loci that affect DTH in Hokkaido.

404 The sequenced positions (based on Nipponbare) were selected using known

405 polymorphisms among varieties in Hokkaido that were observed in previous studies

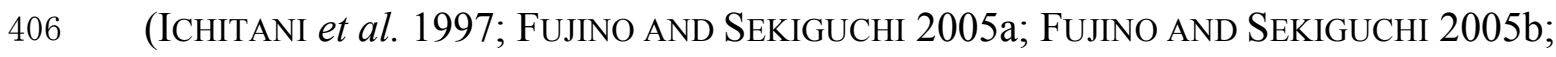

407 NonOue et al. 2008; FujINO et al. 2013; Koo et al. 2013). Hd1 showed multiple

408 differences between A58 and Kitaake; Kitaake possesses a functional allele that is also

409 found in Nipponbare. In DTH8, a 19-bp segment (indicated by a rectangle) was deleted

410 in most of the other Hokkaido varieties, but not in Nipponbare, A58, and Kitaake, from

411 which we could not detect any polymorphisms. For Ghd7 and OsPRR37, SNPs

412 observed in Nipponbare and the other two varieties are indicated by boxes.

414 Figure 4 Chromosomal positions of five SNP clusters. 
415 Physical map positions of each SNP detected by ddRAD-Seq are shown by horizontal

416 bars in each chromosome. Positions of SNP clusters that showed significant differences

417 in allele frequency between early- and late-heading populations are indicated by vertical

418 bars on the right side of each chromosome.

$420 \quad$ Figure 5 Epistatic interaction between SNPs on chromosomes 1 and 10 on DTH

421 observed in 2015 and 2016.

422 Average DTH values for the four combinations of genotypes with central SNPs

423 (C1_2301 and C10_43613) in the clusters on Chs 1 and 10, which are indicated by

424 squares. The case of Chs 1 and 10 were selected from all the combinations with Chs 1, 4,

4256 and 10 (Figure S4). When the A58 SNP on Ch 10 (black line) and Kitaake SNP on Ch

42610 (gray line) were respectively coupled with the different parental SNPs, epistatic

427 (allelic) interactions occurred; in particular, the combination of the A58 allele on Ch 1

428 and Kitaake allele on Ch 10 resulted in the shortest DTH.

$430 \quad$ Figure 6 Phenotypic relationships with combinations of six marker genotype values.

431 Among the $30 \mathrm{~F} 4$ lines, nine retained the homozygous alleles in the six loci that corresponded to the SNP clusters with QTLs for DTH and Hd1. The effect of each locus 
on DTH was weighted according to marker genotype values (see Materials and

Methods) based on DTH in 2016. Larger values indicate a stronger effect on DTH.

Empty squares indicate shorter DTH effects relative to black squares. Kitaake contained to 79.0 days. Each marker name indicates the central SNP in the cluster.

Figure 7 Model of different segregation patterns that occurred in the F2 populations derived from two parental combinations of proximal and distal DTH phenotypes.

443 The left panel represents the segregation pattern of the F2 population between parent-a

444 and -b with proximal DTH phenotypes due to the similar genotypes with a few

445 differences. Because of differences in a few alleles with minor effects on DTH, the F2

446 progenies produced transgressive phenotypes. The right panel represents the F2

447 population produced by parents with distal phenotypes and opposite genotypes shows

448 intermediate segregation between both parents. Most of the F2 progenies with mixed

449 genotypes of the parental alleles did not have DTH phenotypes that exceeded those of the parental phenotypes. There are seven loci involved in DTH, and their effects on 
451 DTH are ordered as $1>>>7 . \mathrm{S}$ and L indicate the effect of an allele at each locus that

452 makes DTH shorter or longer, respectively.

454 Figure S1 Experimental scheme of this study. ddRAD-Seq was carried out to detect

455 differences in allele frequency of genome-wide SNPs between early- and late-heading

$456 \quad$ F4 lines.

Figure S2 Frequency distribution of DTH in the F3 population derived from the A58 $\times$

$459 \quad$ Kitaake cross.

$461 \quad$ Figure S3 Effect of the Ch 4 SNP cluster on DTH in 2015 and 2016.

462 Average DTH values of F4 lines with homozygous A58- and Kitaake-type alleles of the

463 SNP cluster on Ch 4, which is represented by the central SNP in the cluster, C4_19396.

464 Significant differences $(\mathrm{P}<0.01)$ between average DTH values were observed in both $465 \quad 2015$ and 2016.

Figure S4 Genetic interactions between SNP clusters on Chs 1, 4, 6, and 10 relative to 
469 Average DTH values (y-axis) of F4 lines with each genotype are shown in squares. Six

470 combinations of any two loci among the SNP clusters on Chs 1, 4, 6 and 10 are depicted.

471 A58-type alleles (black line) and Kitaake-type alleles (gray line) are paired with another

472 locus corresponding to A58-type alleles (the left side) or Kitaake-type alleles (the right

473 side) in $\mathrm{x}$-axis, respectively. An allelic interaction (non-additive interaction) was

474 detectable where the black line crossed the gray line.

\section{Literature Cited}

Alonso-Blanco, C., and B. Mendez-Vigo, 2014 Genetic architecture of naturally 
Bolger, A. M., M. Lohse and B. Usadel, 2014 Trimmomatic: a flexible trimmer for

Brambilla, V., J. Gomez-Ariza, M. Cerise and F. Fornara, 2017 The Importance of Cereals. Frontiers in Plant Science 8. G3-Genes Genomes Genetics 1: 171-182. in an Interspecific Tomato Cross. Genetics 134: 585-596. Monsters. Evol Biol 40: 310-315. major genetic factors generating naturally occurring variation in heading date among Asian rice cultivars. Theoretical and Applied Genetics 122: 1199-1210.

Fujino, K., and H. Sekiguchi, 2005a Mapping of QTLs conferring extremely early 
heading in rice (Oryza sativa L.). Theoretical and Applied Genetics 111:

$$
393-398 .
$$

Fujino, K., and H. Sekiguchi, 2005b Identification of QTLs conferring genetic variation for heading date among rice varieties at the northern-limit of rice cultivation. Breeding Science 55: 141-146.

Fujino, K., U. Yamanouchi and M. Yano, 2013 Roles of the Hd5 gene controlling heading date for adaptation to the northern limits of rice cultivation. Theoretical and Applied Genetics 126: 611-618.

Goddard, M. E., and B. J. Hayes, 2007 Genomic selection. Journal of Animal Breeding and Genetics 124: 323-330.

Hagiwara, W. E., K. Onishi, I. Takamure and Y. Sano, 2006 Transgressive segregation due to linked QTLs for grain characteristics of rice. Euphytica 150: 27-35.

Harlan, J. R., 1976 Genetic Resources in Wild Relatives of Crops. Crop Science 16: 329-333.

Hori, K., K. Matsubara and M. Yano, 2016 Genetic control of flowering time in rice: integration of Mendelian genetics and genomics. Theoretical and Applied 

Studies in Crop Plants. Annual Review of Plant Biology, Vol 65 65: 531-551.

Ichitani, K., Y. Okumoto and T. Tanisaka, 1997 Photoperiod sensitivity gene of Se-1 locus found in photoperiod insensitive rice cultivars of the northern limit region of rice cultivation. Breeding Science 47: 145-152. Strains. Plant Physiology 164: 671-682. confer the photoperiodic control of flowering in rice (a short-day plant). Plant Journal 22: 391-399. Wide Range of Latitudes. Molecular Plant 6: 1877-1888. 

panicle branch initiation. Proceedings of the National Academy of Sciences of the United States of America 95: 1979-1982.

Langmead, B., and S. L. Salzberg, 2012 Fast gapped-read alignment with Bowtie 2. Nature Methods 9: 357-U354. of quantitative trait loci controlling extremely early heading in rice. Theoretical and Applied Genetics 116: 715-722. genetic interaction associated with an extreme phenotype using assorted F2 populations in rice. Molecular Breeding 33: 997-1003. Genotyping in Model and Non-Model Species. Plos One 7. 
Rieseberg, L. H., M. A. Archer and R. K. Wayne, 1999 Transgressive segregation, adaptation and speciation. Heredity 83: 363-372. is the primary cause of phenotypic diversification. Proceedings of the National Academy of Sciences of the United States of America 99: 12242-12245. architecture necessary for transgressive segregation is common in both natural and domesticated populations. Philosophical Transactions of the Royal Society of London Series B-Biological Sciences 358: 1141-1147.

Takata, M., Y. Kishima and Y. Sano, 2005 DNA methylation polymorphisms in rice $57-63$. genetic potential from the wild. Science 277: 1063-1066. 

Crosses of Barley. Euphytica 29: 585-594. in Ghd7 is an important regulator of heading date and yield potential in rice. Nature Genetics 40: 761-767.

574 Yano, M., Y. Katayose, M. Ashikari, U. Yamanouchi, L. Monna et al., 2000 Hd1, a major photoperiod sensitivity quantitative trait locus in rice, is closely related to the arabidopsis flowering time gene CONSTANS. Plant Cell 12: 2473-2483. 
Table 1 The effect of Hd1 locus on days to heading in F2 population derived from A58 x Kitaake cross

\begin{tabular}{|c|c|c|c|c|c|c|c|c|c|}
\hline \multirow{2}{*}{$\begin{array}{l}\text { Marker } \\
\text { name }\end{array}$} & \multirow[b]{2}{*}{ Chr. } & \multirow[b]{2}{*}{ Position } & \multicolumn{3}{|c|}{ No. of plants } & \multicolumn{3}{|c|}{ Average DTH } & \multirow[b]{2}{*}{$P$} \\
\hline & & & A58-type & Heterozygous & Kitaake-type & A58-type & Heterozygous & Kitaake-type & \\
\hline Hd1 & 6 & & 56 & 103 & 73 & 81.3 & 79.5 & 78.8 & $4 \times 10^{-4}$ \\
\hline
\end{tabular}


Table 2 The effect of SNP clusters on days to heading in F4 lines derived from the cross between A58 and Kitaake

\begin{tabular}{|c|c|c|c|c|c|c|c|c|}
\hline \multirow[b]{2}{*}{ Marker name } & \multirow[b]{2}{*}{ Chr. } & \multirow[b]{2}{*}{ Position } & \multicolumn{3}{|c|}{ DTH (2015) } & \multicolumn{3}{|c|}{ DTH (2016) } \\
\hline & & & A58-type & Kitaake-type & $P$ & A58-type & Kitaake-type & $P$ \\
\hline C1_2292 & 1 & $25,250,821$ & $68.5 \pm 2.02$ & $73.6 \pm 1.78$ & 0.07856 & $75.9 \pm 1.72$ & $79.3 \pm 1.72$ & 0.1745 \\
\hline C1_2301 & 1 & $25,270,205$ & $68.5 \pm 2.02$ & $73.6 \pm 1.78$ & 0.07856 & $75.9 \pm 1.72$ & $79.3 \pm 1.72$ & 0.1745 \\
\hline C1_2745 & 1 & $28,608,975$ & $68.1 \pm 1.85$ & $73.4 \pm 1.54$ & $0.03627 *$ & $75.3 \pm 1.59$ & $79.0 \pm 1.52$ & 0.1095 \\
\hline C1_2968 & 1 & $30,128,355$ & $66.9 \pm 1.96$ & $72.7 \pm 1.66$ & $0.03616 *$ & $74.1 \pm 1.94$ & $78.1 \pm 1.46$ & 0.1195 \\
\hline C2_10782 & 2 & 790,734 & $68.1 \pm 1.83$ & $71.8 \pm 1.59$ & 0.139 & $74.8 \pm 1.65$ & $78.1 \pm 1.47$ & 0.1461 \\
\hline C2_10784 & 2 & 790,866 & $68.1 \pm 1.83$ & $71.8 \pm 1.59$ & 0.139 & $74.8 \pm 1.65$ & $78.1 \pm 1.47$ & 0.1461 \\
\hline C4_19210 & 4 & $29,823,605$ & $74.8 \pm 1.14$ & $66.6 \pm 1.61$ & $0.0003583 * * *$ & $81.9 \pm 0.65$ & $73.7 \pm 1.34$ & $0.000012 * * *$ \\
\hline C4_19304 & 4 & $30,511,751$ & $76.3 \pm 1.03$ & $66.1 \pm 1.61$ & $0.0000237 * * *$ & $82.1 \pm 0.72$ & $73.2 \pm 1.43$ & $0.0000162 * * *$ \\
\hline C4_19390 & 4 & $31,264,195$ & $76.9 \pm 1.08$ & $65.1 \pm 1.39$ & $1.13 \mathrm{E}-06 * * *$ & $82.5 \pm 0.74$ & $72.5 \pm 1.3$ & $1.07 \mathrm{E}-06 * * *$ \\
\hline C4_19586 & 4 & $32,438,249$ & $75.5 \pm 1.21$ & $65.7 \pm 1.43$ & $0.0000955 * * *$ & $81.6 \pm 0.68$ & $72.9 \pm 1.31$ & $9.34 \mathrm{E}-06 * * *$ \\
\hline C6_27238 & 6 & $24,515,347$ & $66.5 \pm 1.72$ & $70.3 \pm 1.91$ & 0.2326 & $73.5 \pm 1.78$ & $76.6 \pm 1.62$ & 0.1031 \\
\hline C6_27270 & 6 & $24,574,372$ & $71.9 \pm 1.64$ & $65.7 \pm 2.03$ & $0.02257 *$ & $77.2 \pm 1.78$ & $73.8 \pm 1.51$ & 0.1675 \\
\hline C6_27338 & 6 & $24,978,329$ & $71.1 \pm 1.78$ & $66.6 \pm 2.16$ & 0.09858 & $76.5 \pm 1.67$ & $75.1 \pm 1.92$ & 0.5787 \\
\hline C6_27340 & 6 & $24,985,031$ & $71.3 \pm 1.57$ & $65.2 \pm 2.24$ & $0.04015 *$ & $76.9 \pm 1.53$ & $73.3 \pm 1.74$ & 0.178 \\
\hline C6_27365 & 6 & $25,230,125$ & $71.7 \pm 1.67$ & $66.0 \pm 2.21$ & 0.0532 & $77.6 \pm 1.59$ & $74.5 \pm 1.67$ & 0.1907 \\
\hline C6_27415 & 6 & $25,515,786$ & $71.5 \pm 1.67$ & $66.9 \pm 2.03$ & 0.1137 & $77.3 \pm 1.59$ & $75.1 \pm 1.51$ & 0.3546 \\
\hline C10_43613 & 10 & 593,246 & $71.9 \pm 1.68$ & $64.4 \pm 1.93$ & $0.008726 * * *$ & $77.3 \pm 1.42$ & $72.1 \pm 1.9$ & $0.04205 *$ \\
\hline C10_43829 & 10 & 709,525 & $72.5 \pm 1.4$ & $65.7 \pm 2.28$ & $0.022 *$ & $78.1 \pm 1.22$ & $73.3 \pm 2.42$ & 0.09918 \\
\hline C10_43830 & 10 & 709,622 & $72.8 \pm 1.47$ & $65.7 \pm 2.06$ & $0.01105 *$ & $78.2 \pm 1.3$ & $73.5 \pm 2.2$ & 0.08329 \\
\hline
\end{tabular}




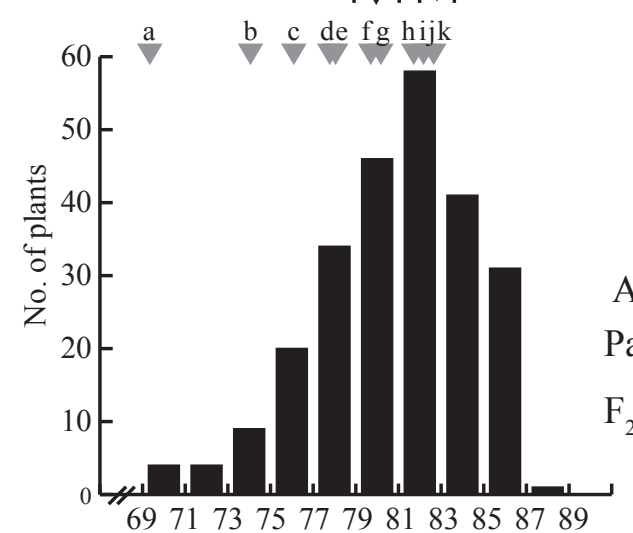

A58 - Kitaake

Parental difference: 0.7 days

$\mathrm{F}_{2}$ diffenrence: 18.0 days

6971737577798183858789

$\underset{\mathrm{F}_{2}}{\stackrel{\text { difference }\left(\mathrm{F}_{2} \max -\mathrm{F}_{2} \min \right)}{\longrightarrow}}$

Transgressive index : 25.7

( $\mathrm{F}_{2}$ difference / Parental difference)

$\mathrm{B}$

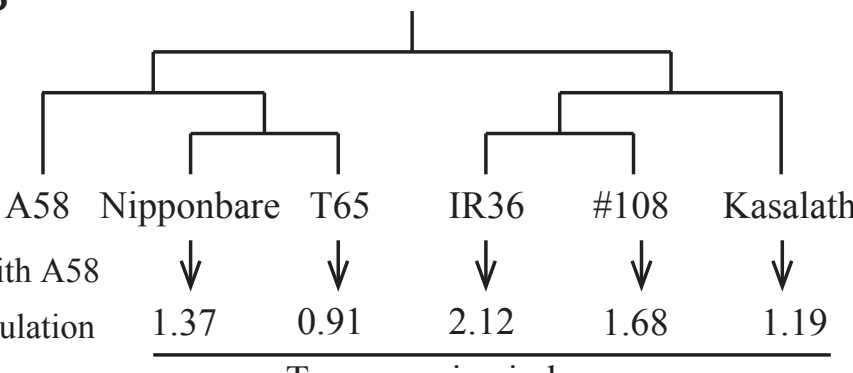

Transgressive index

Figure 1 Transgressive segregation and genetic relationships between parental varieties.

(A) Frequency distribution of DTH from A58 $\times$ Kitaake F2 plants. The transgressive index represents the ratio of the of the F2 population DTH distribution to the parental difference. The DTH difference between A58 and Kitaake was 0.7 days, and the DTH range in the F2 population was 18.0 days, which resulted in a transgressive index of 25.7. Standard DTH values of 11 varieties in Hokkaido are indicated by gray arrowheads: (a) Kitaibuki, (b) Hakucho-mochi, (c) Daichinohoshi (d) Hatsushizuku, (e) Hoshinoyume, (f) Kuiku180, (g) Hokuiku-mochi, (h) Nanatsuboshi, (i) Kirara397, (j) Hoshimaru, and (k) Gimpu. (B) Transgressive indexes of crosses between A58 and each of five other varieties. The phylogenetic relationships and the associated dendrogram for the five O. sativa varieties, Nipponbare (japonica), T65 (japonica), IR36 (indica), \#108 (indica), and Kasalath (indica, Aus), are presented based on information provided in TAKATA et al. (2005). To calculate the transgressive index, DTH of parental varieties and F2 plants were calculated based on data from OTA et al. (2014). 

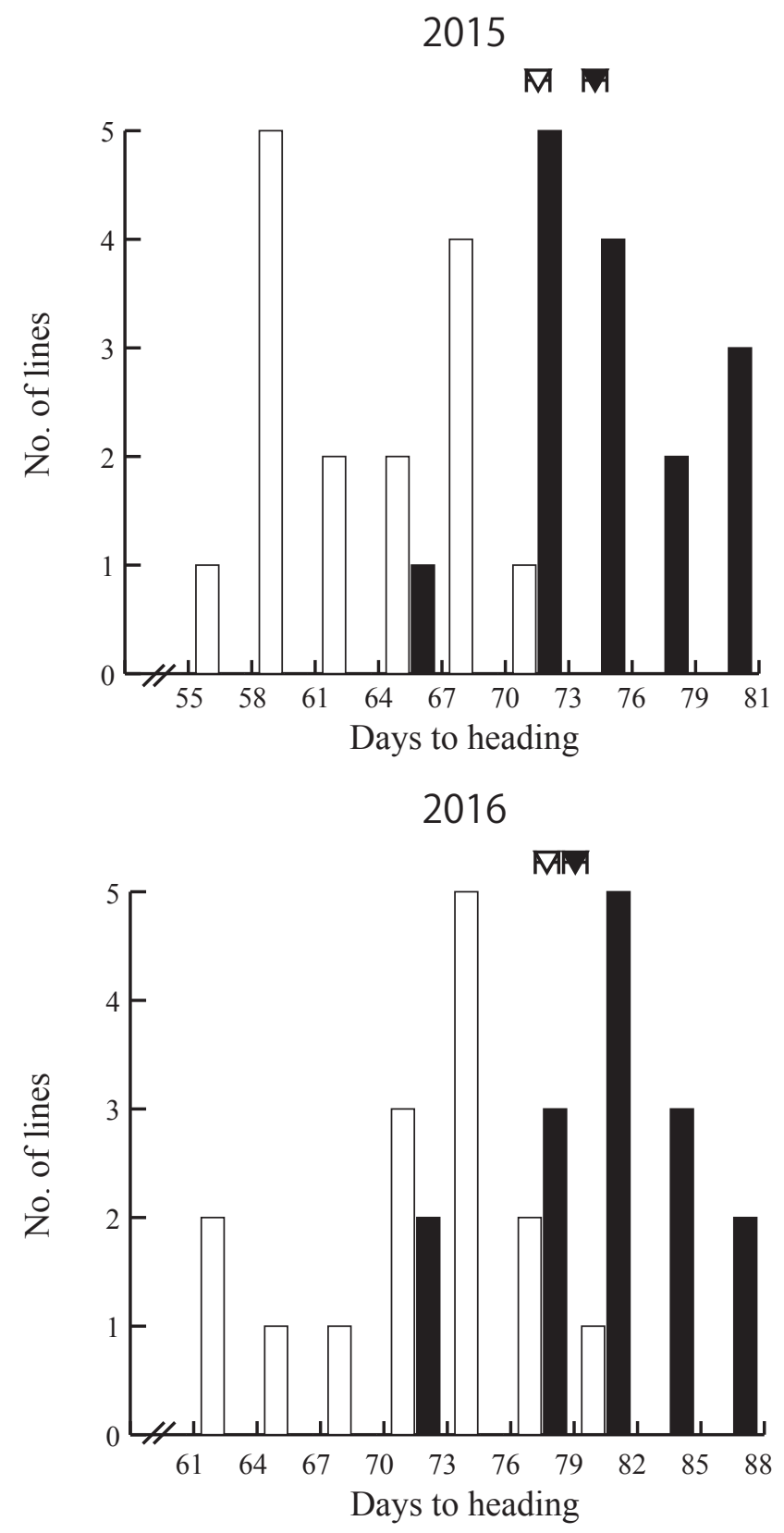

Figure 2 Frequency distribution of DTH in early- and late-heading F4 lines derived from the A58 $\times$ Kitaake cross in 2015 and 2016.

DTH of the 15 early- and 15 late-heading F4 lines selected in the F3 population was examined in the two years, 2015 and 2016. Early- and late-heading lines are indicated by white and black, respectively. Kitaake and A58 DTH are indicated by white and black arrowheads, respectively, with bars indicating S. E. 


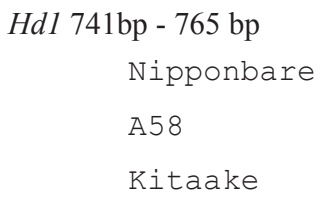
DTH8 $161 \mathrm{bp}-210 \mathrm{bp}$ Nipponbare A58
Kitaake

Ghd7 341bp - 390 bp Nipponbare

A5 8

Kitaake

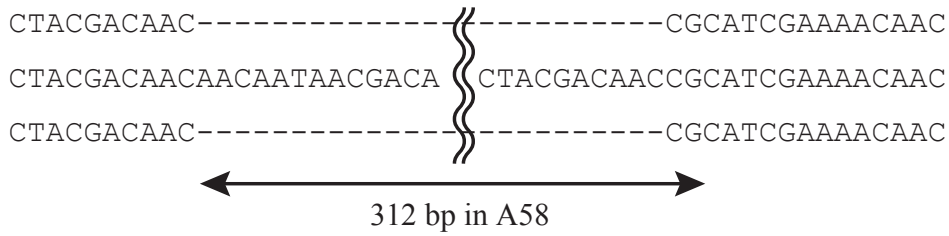

GCGAGGCGGCGGCGGCGGCTGCGG CTGGCGGCGGCGGCTGCGG GAGCAGC GCGAGGCGGCGGCGGCGGCTGCGGCTGGCGGCGGCGGCTGCGGGAGCAGC GCGAGGCGGCGGCGGCGGCTGCGGCTGGCGGCGGCGGCTGCGGGAGCAGC

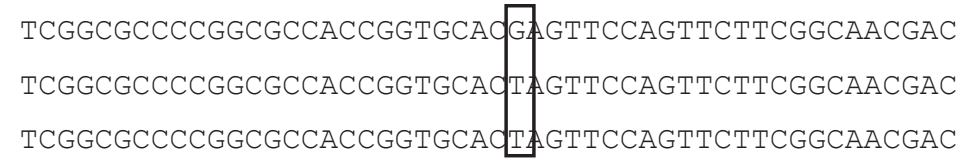

OsPRR37 2571bp - $2620 \mathrm{bp}$ $\begin{array}{ll}\text { Nipponbare } & \text { GTGCGGTACCAGAGCAGAAAGAGGTTFGCCAGCAGCGGCCAAGGGTCCG } \\ \text { A58 } & \text { GTGCGGTACCAGAGCAGAAAGAGGCFGCCGAGCAGCGGCCAAGGGTCCG } \\ \text { Kitaake } & \text { GTGCGGTACCAGAGCAGAAAGAGGCEGCCGAGCAGCGGCCAAGGGTCCG }\end{array}$

Figure 3 Comparisons of partial nucleotide sequences from Nipponbare, A58, and Kitaake for the four major loci that affect DTH in Hokkaido.

The sequenced positions (based on Nipponbare) were selected using known polymorphisms among varieties in Hokkaido that were observed in previous studies (ICHITANI et al. 1997; FUJINO AND SEKIGUCHI 2005a; FUJINO AND SEKIGUCHI 2005b; NONOUE et al. 2008; FUJINO et al. 2013; KOO et al. 2013). Hd1 showed multiple differences between A58 and Kitake; Kitaake possesses a functional allele that is also found in Nipponbare. In DTH8, a 19-bp segment (indicated by a rectangle) was deleted in most of the other Hokkaido varieties, but not in Nipponbare, A58, and Kitaake, from which we could not detect any polymorphisms. For Ghd7 and OsPRR37, SNPs observed in Nipponbare and the other two varieties are indicated by boxes. 
Rice chromosomes

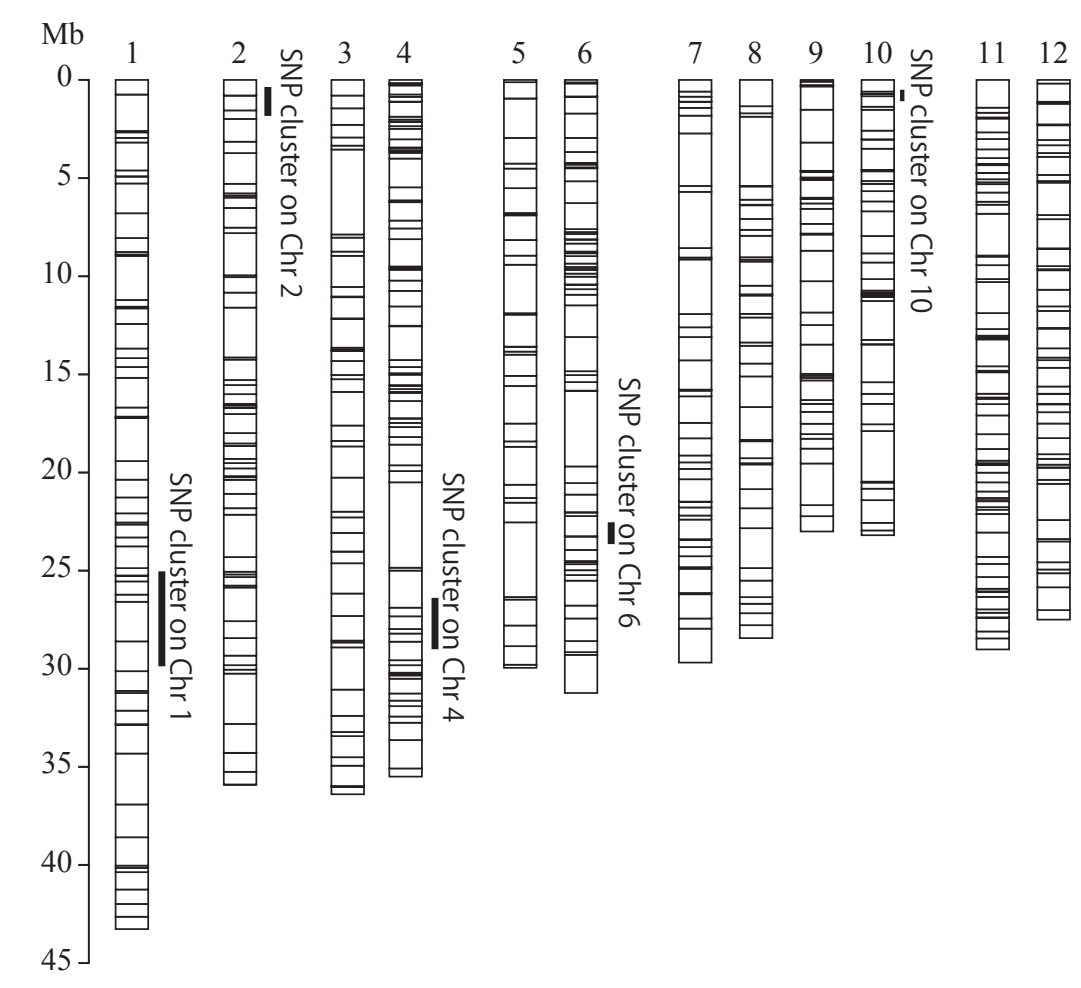

Figure 4 Chromosomal positions of five SNP clusters.

Physical map positions of each SNP detected by ddRAD-Seq are shown by horizontal bars in each chromosome. Positions of SNP clusters that showed significant differences in allele frequency between early- and late-heading populations are indicated by vertical bars on the right side of each chromosome. 

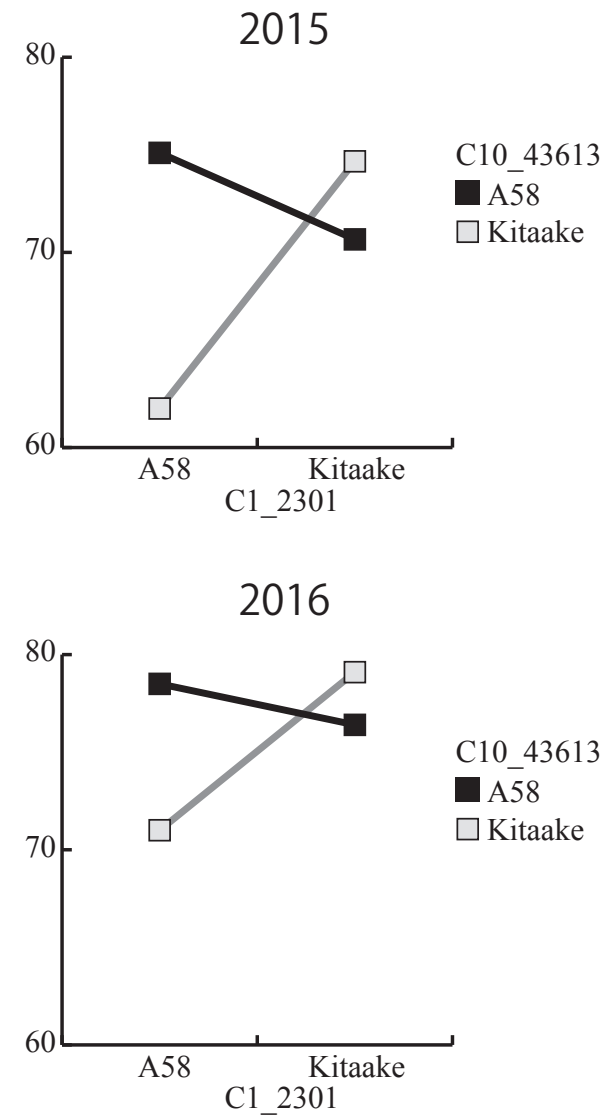

Figure 5 Epistatic interaction between SNPs on chromosomes 1 and 10 on DTH observed in 2015 and 2016.

Average DTH values for the four combinations of genotypes with central SNPs (C1_2301 and C10_43613) in the clusters on Chs 1 and 10, which are indicated by squares. The case of Chs 1 and 10 were selected from all the combinations with Chs 1, 4, 6 and 10 (Figure S5). When the A58 SNP on Ch 10 (black line) and Kitaake SNP on Ch 10 (gray line) were respectively coupled with the different parental SNPs, epistatic (allelic) interactions occurred; in particular, the combination of the A58 allele on Ch 1 and Kitaake allele on Ch 10 resulted in the shortest DTH. 


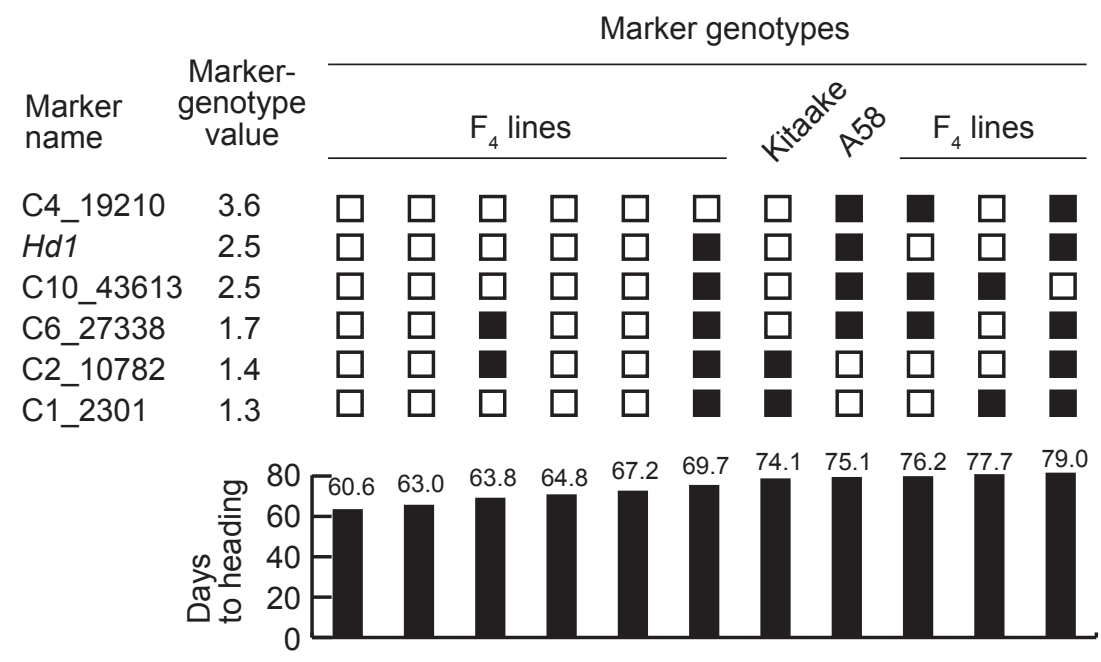

Figure 6 Phenotypic relationships with combinations of six marker genotype values.

Among the 30 F4 lines, nine retained the homozygous alleles in the six loci that corresponded to the SNP clusters with QTLs for DTH and Hd1. The effect of each locus on DTH was weighted according to marker genotype values (see Materials and Methods) based on DTH in 2016. Larger values indicate a stronger effect on DTH. Empty squares indicate shorter DTH effects relative to black squares. Kitaake contained four shorter DTH alleles in Ch 4, Hd1, Ch 10, and Ch 6, whereas A58 possessed two shorter DTH alleles in Ch 2 and Ch 1. The two parental cultivars, Kitaake and A58, had DTH of 74.1 and 75.1 days, respectively. DTH in the selected F4 lines ranged from 60.6 to 79.0 days. Each marker name indicates the central SNP in the cluster. 


\section{Proximal parental DTH phenotypes}

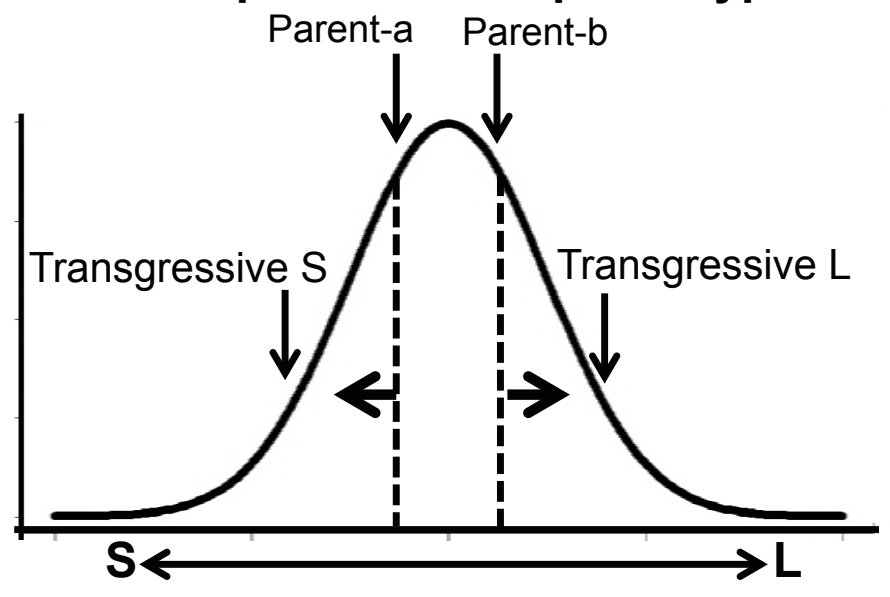

Distal parental DTH phenotypes

\begin{tabular}{llllllll} 
QTLS & 1 & 2 & 3 & 4 & 5 & 6 & 7 \\
\hline Parent-a & S & S & S & S & S & L & L \\
Parent-b & S & S & S & S & L & S & S
\end{tabular}

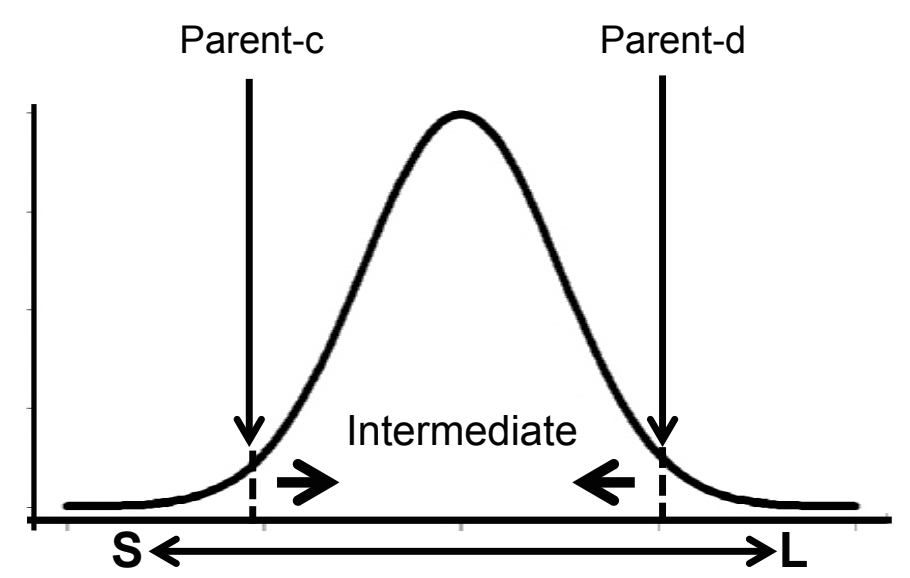

Transgressive short DTH

$\begin{array}{lllllll}\text { Line-A } & \text { S S S S S S S } \\ \text { Line-B } & \text { S S S S S S L } \\ \text { Line-C } & \text { S S S S S L S }\end{array}$

Line-C S S S S I S

Transgressive long DTH

Line-D $S$ S $S$ S $S I$

Line-E $S$ S S S L I S

Line-F S S S S L L L

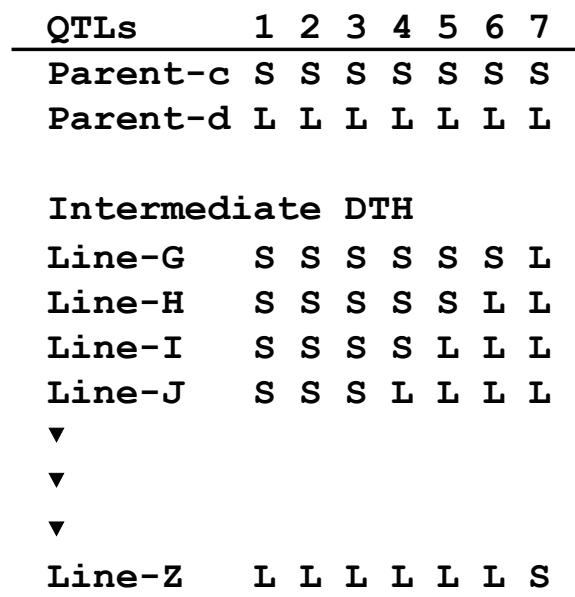

Effect of QTL $1>2>3>4>5>6>7$

Figure 7 Model of different segregation patterns that occurred in the F2 populations derived from two parental combinations of proximal and distal DTH phenotypes.

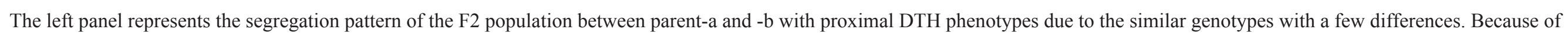

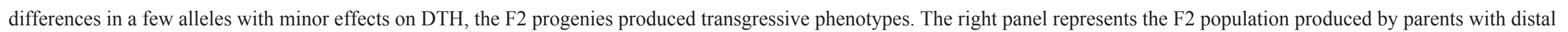
phenotypes and opposite genotypes shows intermediate segregation between both parents. Most of the F2 progenies with mixed genotypes of the parental alleles did not have DTH

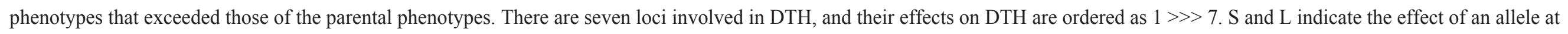
each locus that makes DTH shorter or longer, respectively. 